\title{
ESTUDIO DE UN DISEÑO DIDÁCTICO EN UN CURSO DESTINADO A LA FORMACIÓN DE PROFESORES DE FÍSICA BÁSICA
}

\author{
CÁRDENAS, MARTA y RAGOUT DE LOZANO, SILVIA \\ Departamento de Física. Facultad de Ciencias Exactas y Tecnología \\ Universidad Nacional de Tucumán. Av. Independencia, 1800. 4000 SM de Tucumán. Argentina \\ E-mail: macar@csnat.unt.edu.ar - sragout@ herrera.unt.edu.ar
}

\begin{abstract}
SUMMARY
We study a proposal made by Duschl in regard to the use of Laudan's triadic network concerning the relationship between the theory, method and objectives of Science, as well as Shapere's observation model, for didactic purposes. We analyse the possible adaptation of the above theoretical models to fulfill the requirements of the Argentinian formal education. Both epistemological systems, adequate for facilitating the conceptual changes in the classroom, were taken as referents for the design of teaching approaches of thermal phenomena in Physics.

We describe the way in which we deal with certain specific subjects in one particular course, addressed to Physics teachers, in which the above design was used. The results obtained are analyzed and dicussed and certain still-standing issues are mentioned.
\end{abstract}

\section{INTRODUCCIÓN}

En este artículo se presenta el diseño de un curso de postgrado, se relata su puesta en práctica y se analizan algunos aspectos que nos parecieron importantes durante el desarrollo y la evaluación del mismo. El curso forma parte del currículo del Magister en Enseñanza de las Ciencias (Área Física), carrera de postgrado de la Universidad Nacional de Tucumán (Argentina). Esta carrera de postgrado exige como requisito de admisión tener un título de grado que habilite para la docencia en física y antecedentes de actividad docente en la disciplina.

Los cursos del Magister en Enseñanza de las Ciencias abarcan tres áreas:

Área I: Aportes de las ciencias psicosociales y de la investigación educativa en ciencias a la enseñanza de las ciencias experimentales.
Área II: Actualización en la disciplina específica (física).

Área III: Integración de las áreas I y II en contextos educativos específicos.

El curso que desarrollamos, «La física de los fenómenos térmicos: su enseñanza en distintos niveles de la educación formal», corresponde al área III y la planificación del mismo se hizo en función de tres objetivos específicos:

a) Revisar y profundizar contenidos de la física.

b) Presentar un modelo de diseño curricular aplicable en diferentes niveles del sistema educativo. 
c) Transferir la propuesta curricular a través de una experiencia en la cual los profesores participantes, desde su rol de alumnos del curso, tuvieran la oportunidad de experimentar in situ las implicaciones de la propuesta, para después implementarla en su práctica docente

El modelo de diseño desarrollado está fundamentado desde lo epistemológico en las ideas de Larry Laudan sobre la estructura y el progreso de la ciencia, y desde la psicología del aprendizaje en la perspectiva del cambio conceptual.

\section{LA PROPUESTA METODOLÓGICA DE DUSCHL}

En el libro Renovar la enseñanza de las ciencias, Duschl (1997) sostiene la tesis de que un análisis del desarrollo de las teorías científicas puede orientar las decisiones del profesor sobre qué contenidos seleccionar a la hora de planificar el currículo de un curso de ciencia. Duschl parte del supuesto de que los procedimientos empleados en la enseñanza y el aprendizaje de las ciencias son más efectivos si hay similitud entre los procedimientos empleados en el diseño instruccional y los que se desea que los alumnos desarrollen y adquieran durante el aprendizaje.

La ciencia es una disciplina que busca comprender el cómo y el por qué de los fenómenos, y lo que se persigue en un curso de física es que los estudiantes sean capaces de explicar el cómo y el por qué de los fenómenos del mundo físico: por lo tanto, lo que tratamos es que nuestros alumnos aprendan a realizar actividades similares a las que realiza el científico en su afán de acrecentar el conocimiento.

Duschl (1998) sostiene que los profesores pueden emplear en la planificación de sus cursos las pautas metodológicas que los epistemólogos proponen para explicar la estructura y la reestructuración de las teorías científicas.

El estudio realizado por los filósofos e historiadores de la ciencia sobre cómo se producen los cambios de teoría ha inducido a los psicólogos y a los investigadores en didáctica de la ciencia (Posner et al., 1982), a sugerir que es válido y útil establecer un paralelismo entre los principios que rigen los cambios de teorías en ciencia y los procesos cognitivos que emplean los alumnos en el aprendizaje de la ciencia. Es oportuno destacar, entonces, que la aplicación de las teorías del cambio conceptual en la educación es fruto de las ideas de los epistemólogos acerca del carácter provisorio del conocimiento científico.

Así como Piaget desarrolló la epistemología genética a partir del análisis en profundidad del desarrollo de las estructuras cognitivas de los niños, la propuesta de Duschl es emplear un proceso inverso consistente en aplicar a la didáctica de la ciencia los esquemas epistemológicos de su desarrollo y progreso.
Los esquemas epistemológicos del desarrollo de la ciencia fundamentan modelos actuales de aprendizaje de la ciencia y, desde un punto de vista estructural, puede establecerse un paralelismo entre teorías científicas y esquemas cognitivos (Tabla I).

Tabla I

\begin{tabular}{|ll|}
\hline TEORÍAS CIENTÍfICAS & ESQUEMAS COGNITIVOS \\
\hline Red de hechos, principios & Grupo de conceptos \\
y leyes que se unen & y proposiciones, gobernados \\
mediante prácticas & por las leyes de la lógica \\
metodológicas y metas & y los valores sociales, \\
comunes de indagación. & que juntos guían las síntesis \\
& del conocimiento. \\
\hline
\end{tabular}

Desde el punto de vista histórico, en la ciencia -y también en su enseñanza- se ha privilegiado el tratamiento del contexto de justificación, relegando el contex to de descubrimiento. De manera concomitante, en la educación se excluyen en las clases de ciencia los aspectos referidos a refinamientos, ajustes y cambios de teoría, dando una imagen de la ciencia como producto acabado.

El análisis histórico del desarrollo del conocimiento científico muestra que el mismo pasa por períodos de consenso y de desacuerdo, y que los cambios de teoría no son en general globales sino de carácter gradual. Sin embargo, el modelo epistemológico ciencia normal ciencia revolucionaria de Kuhn (1978) fue el inspirador del primer modelo de cambio conceptual (Posner et al., op.cit.). El principal defecto de esta fundamentación fue suponer que los cambios de teoría desencadenan automáticamente cambios en los métodos y objetivos de investigación (modelo holístico).

Por su parte, Laudan (1977) sostiene que el modelo de progreso científico es concebible como un cambio en las «tradiciones de investigación», que son conjuntos de creencias acerca de las clases de identidades y procesos que integran el dominio de la investigación, más un conjunto de normas epistemológicas y metodológicas acerca de cómo se debe investigar ese dominio, cómo se debe poner a prueba la teoría, cómo se debe recabar datos, y similares.

Laudan afirma, en oposición a lo sostenido por Kuhn, que el cambio de teoría es menos holístico y más gradual, y que los cambios en las teorías, métodos y objetivos de la ciencia pueden ocurrir -y ocurren- por separado en distintos períodos, de modo que un cambio en el compromiso con la teoría no implica necesariamente un cambio simultáneo en los compromisos con los métodos y los objetivos.

De manera análoga, Villani (1992) argumenta que el aprendizaje de las ciencias puede ser descrito como un proceso que incluye no sólo cambios en las ideas de los 
Figura 1

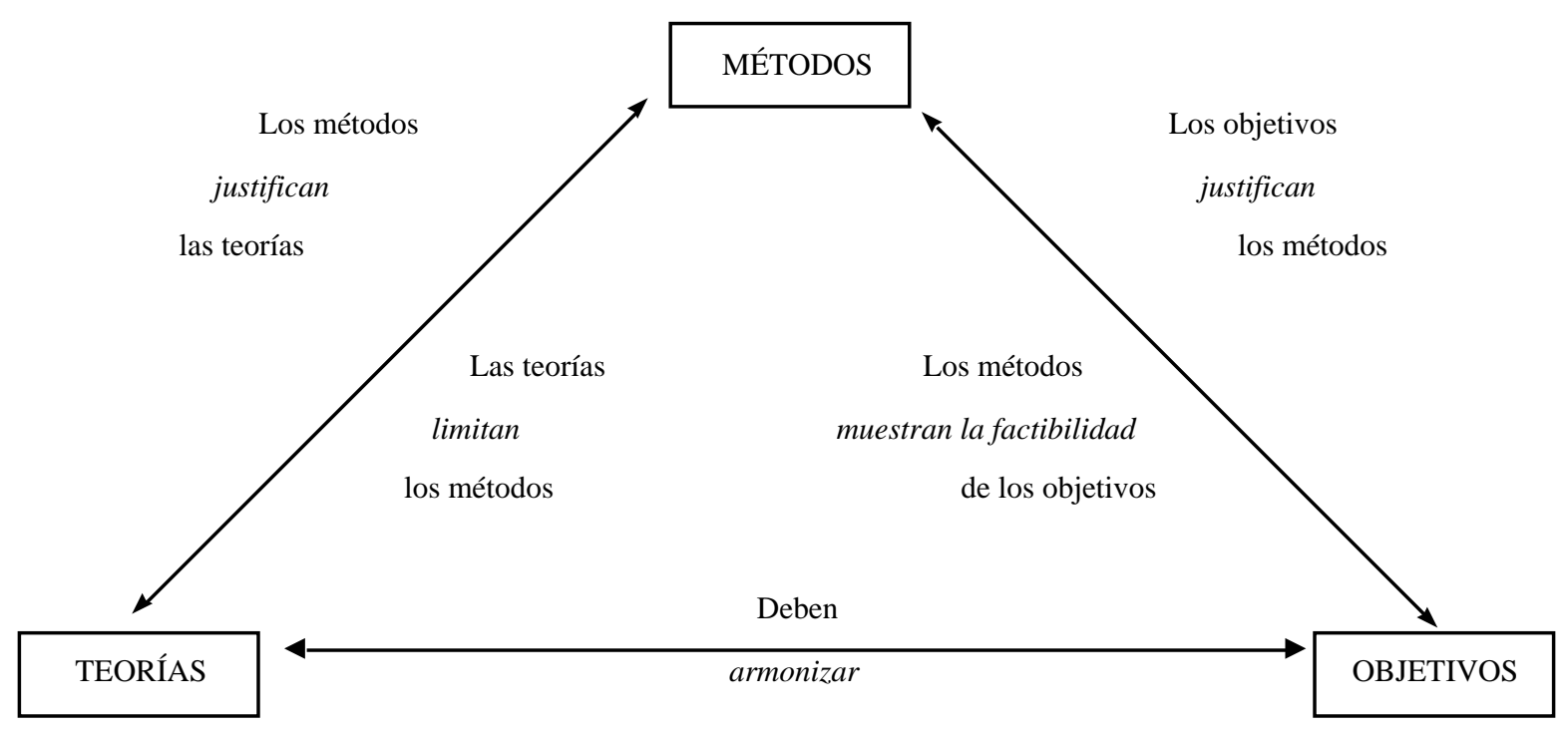

aprendices o la aceptación de nuevas concepciones, sino principalmente cambios en la naturaleza de las preguntas, en las entidades básicas, en los métodos y en los objetivos perseguidos en el aprendizaje.

También Gil Pérez (1986) destaca que dar una imagen deformada de la ciencia, como producto acabado, genera problemas en el aula y hace necesaria la emergencia de un nuevo paradigma de enseñanza-aprendizaje. Como alternativa propone un modelo de aprendizaje de la ciencia como investigación, en una perspectiva de cambio conceptual y metodológico.

En función de las coincidencias que venimos destacando, nos parece apropiado exponer algunos de los aspectos de la propuesta de Duschl (1997, op. cit.), con los que coincidimos.

Duschl discute, entre otros, dos modelos que describen los procesos de cambio de teorías: $a$ ) la red triádica de Laudan; y $b$ ) el proceso de observación tripartita de Shapere. Son modelos epistemológicos para explicar o caracterizar el desarrollo del conocimiento científico. Este autor muestra de qué manera pueden utilizarse como esquemas de conocimiento procedimental para guiar tanto la enseñanza como el aprendizaje. En los parágrafos siguientes mostraremos las principales características de ambos modelos.

\section{LA RED TRIÁDICA DE LAUDAN}

Como ya se dijo, según Laudan (1984), el desarrollo del conocimiento no está subordinado sólo a los compromi- sos con la teoría, sino que hay tres niveles de compromiso para los científicos:

- con las teorías;

- con las metodologías;

- con los fines u objetivos de la ciencia.

En este modelo, los tres niveles presentan el mismo estatus jerárquico, lo que se puede representar en una red triádica en la que se muestra el carácter no holístico de los cambios del conocimiento científico. En la figura 1 se esquematiza este sistema epistemológico o red triádica de justificación del conocimiento en ciencia, en el que se visualiza que los cambios pueden ocurrir por separado $\mathrm{y}$ en forma fragmentaria, comenzando en cualquiera de los vértices de la red.

Laudan (1984, p. 62) afirma que: «Existe un complejo proceso de ajuste y justificación mutuos entre los tres niveles de compromiso científico [...] El orden implícito en el enfoque jerárquico debe dar paso a una especie de principio de nivelación que subraye la dependencia mutua entre estos tres niveles.»

Duschl destaca que en las clases de ciencia se suele presentar el conocimiento científico en forma holística, dando una imagen acabada que enmascara la estructura temporal y los sucesos cruciales responsables de los cambios, por lo que propone la utilización de la red triádica en la planificación de currículos e intervenciones didácticas, como un «principio de nivelación» que permitiría proporcionar a los alumnos estrategias para la construcción de su propio conocimiento, a la vez que se presentaría la ciencia de manera más significativa, revelando su carácter dinámico. 
En síntesis, la adopción del modelo epistemológico de Laudan implicaría las siguientes consecuencias para la didáctica de las ciencias.

Es necesario enseñar explícitamente a los estudiantes:

- conceptos y teorías;

- reglas metodológicas;

-objetivos y valores científicos.

Duschl, basándose en la red triádica de Laudan, destaca que, para el aprendizaje de la ciencia, el cambio conceptual pretende el establecimiento de concepciones comunes a todos los miembros de la clase, las cuales deben ser coincidentes con las aceptadas por la comunidad científica. Por ello, deberá tenerse en cuenta:

a) Respecto a los conceptos y teorías (Tabla II)

Tabla II

\begin{tabular}{|lll|}
\hline Si entre los alumnos: & El profesor debe: \\
\hline $\begin{array}{l}\text { - Hay desacuerdo sobre } \\
\text { un concepto }\end{array}$ & $\rightarrow$ & $\begin{array}{l}\text { Implementar estrategias } \\
\text { para lograr consenso }\end{array}$ \\
$\begin{array}{l}\text { - Hay consenso, pero el } \\
\text { concepto no es aceptable } \\
\text { científicamente }\end{array}$ & $\rightarrow$ & $\begin{array}{l}\text { Emplear estrategias que } \\
\text { provoquen desacuerdo, } \\
\text { para promover el cambio } \\
\text { conceptual }\end{array}$ \\
\hline
\end{tabular}

b) Respecto a la metodología

A los alumnos les cuesta aceptar y entender los datos científicos si no poseen información relacionada con los procesos de su obtención, procesamiento e interpretación. Consecuentemente, hay que ayudar a los estudiantes a comprender cómo han aprendido a aprender los científicos, ya que saber cómo se buscan soluciones y cómo se identifican nuevas preguntas es un elemento crucial para el desarrollo o apropiación del conocimiento científico.

c) Respecto a los objetivos y metas

Durante el proceso de instrucción:

- Es necesario poner en evidencia que en cada época hay pautas internas y externas que guían la selección de problemas.

- Los estudiantes deben tomar conciencia de que las presiones sociales afectan a la formulación de los objetivos y a la financiación de las investigaciones.

- Los alumnos deben saber diferenciar entre un aspecto básico y uno aplicado de la ciencia y valorar por igual su importancia para el desarrollo del conocimiento científico.

- Se debe resaltar la importancia de las interacciones ciencia-técnica-sociedad en la toma de decisiones respecto a las metas.

\section{EL PROCESO TRIPARTITO DE OBSERVA- CIÓN}

Un aspecto importante para la comprensión de las teorías científicas es analizar la interrelación entre las teorías y el proceso de observación científica.

Shapere (1982) analiza el desarrollo racional de los cambios producidos en la observación científica, destacando que actualmente se resta importancia al papel de los sentidos en la recolección de datos.

Lo que «vemos» depende de lo que «sabemos», por lo que hay diferencia entre «ver cómo» $\mathrm{y}$ «ver qué»:

- «Ver cómo»: Las observaciones se concentran en la descripción literal, sin conocimiento previo.

- «Ver qué»: Las observaciones están asociadas a una teoría, por lo que se hacen con conocimiento previo.

De acuerdo con estas distinciones, la esencia del cambio conceptual es que los alumnos pasen de ser observadores novatos del tipo «ver cómo», a observadores informados de «ver qué».

Shapere sostiene que lo que se denomina observación en ciencia ha experimentado un cambio a lo largo del progreso de la misma: ha pasado de «ver cómo» a «ver qué», y por ello representa una observación como un proceso complejo divido en tres partes:

1) La emisión de información por una fuente.

2) El proceso de transmisión de la información.

3) La recepción de la información.

Este modelo tripartito pone en evidencia que los científicos han construido teorías acerca de la fuente, de la transmisión y del receptor $\mathrm{y}$, cuando se aplica este modelo a observaciones científicas, queda claro que la naturaleza de las pruebas que apoyan (o refutan) las teorías es un producto tanto del conocimiento cognitivo como de los métodos y de los objetivos de las investigaciones: «Hemos aprendido cómo aprender acerca de la naturaleza.» (Duschl, 1997)

En lo que se refiere a la enseñanza de la ciencia, según Duschl, un examen y una articulación de las teorías relativas a la fuente, la transmisión y el receptor ayudan a los estudiantes a comprender la evolución racional de los criterios científicos y a apreciar mejor cómo los científicos han llegado a pensar en la forma que lo hacen 
y a usar los métodos que utilizan. También sostiene que de este modo se pueden proporcionar al estudiante estrategias adecuadas para el aprendizaje significativo de la ciencia.

\section{DESCRIPCIÓN DE LA EXPERIENCIA DI- DÁCTICA}

En función de uno de los objetivos del curso - la planificación de intervenciones didácticas para la enseñanza de los fenómenos térmicos en distintos niveles del sistema educativo- se comenzó con una revisión de los requerimientos de la reforma educativa que se está concretando en nuestro país respecto a los contenidos conceptuales, procedimentales y actitudinales a desarrollar en el tercer ciclo de la enseñanza general básica (EGB 3), la educación polimodal y, también, en los cursos universitarios de física básica.

La reforma educativa mencionada prevé una enseñanza general básica, de carácter obligatorio, conformada por tres ciclos de tres años cada uno: EGB 1 (6 a 8 años), EGB 2 (9 a 11 años) y EGB 3 (12 a 14 años). La educación polimodal (15 a 17 años) no es obligatoria, ofrece cinco modalidades que posibilitan una salida laboral y habilitan para continuar estudios superiores. Todas las modalidades comparten una formación general de fundamento, y cada una de ellas tiene una formación orientada.

Dado que en la reforma educativa se pone particular énfasis en desarrollar el aprendizaje conjunto de contenidos conceptuales, procedimentales y valorativosactitudinales, lo cual puede sintetizarse en saber, saber hacer y saber ser, encontramos que la propuesta de Duschl satisface estos requerimientos, ya que enseñar a los estudiantes:

a) Conceptos y teorías ..................... se corresponde con ......... saber

b) Reglas metodológicas

saber hacer

c) Objetivos y valores científicos

saber ser

Tradicionalmente, el énfasis en la enseñanza de la ciencia ha estado puesto en los contenidos conceptuales. Ahora, en la reforma educativa se jerarquizan los contenidos procedimentales y actitudinales, mostrando la necesidad de que se hagan explícitos y no queden, en el mejor de los casos, dentro del «currículo oculto», y librados al criterio de cada docente. El propósito fundamental de la reforma es fortalecer el núcleo de capacidades fundamentales (intelectuales, metacognitivas, prácticas, interactivas y sociales, etc.) que todos necesitan para su formación integral como personas (saber ser).

En la planificación y el desarrollo del curso que analizamos, se ha tenido en cuenta que la enseñanza y el aprendizaje de los diferentes tipos de contenidos implican diferentes estrategias didácticas y distintos procesos psicológicos (Coll et al., 1994) y, para su articulación, se ha seguido la propuesta de Duschl respecto al uso de la red triádica de Laudan y del proceso tripartito de observación de Shapere.

En la tabla III se muestran los contenidos mínimos sobre fenómenos térmicos, correspondientes a EGB 3 y educación polimodal.

En el nivel universitario básico, los temas referidos a los fenómenos térmicos, desarrollados con la profundidad y rigor formal correspondientes, coinciden en términos generales con los que se muestran en la tabla III como contenidos conceptuales. Respecto a los contenidos procedimentales, las exigencias y posibilidades son obviamente mayores, y por ello requieren una atención especial por parte de los docentes, que muchas veces presuponen que los alumnos poseen competencia en procedimientos básicos, sin que realmente éste sea el caso (Bandiera et al., 1995). Por ello, en el curso que nos ocupa, y después de explicitar detalladamente el referencial teórico adoptado -y adaptado- propuesto por Duschl, se trató de analizar, discutir y diferenciar estrategias instruccionales adecuadas para la enseñanza de estos temas en los distintos niveles educativos.

Se propuso a los participantes la realización de diversas actividades diseñadas en función del marco teórico presentado. Cada actividad se planificó para ser realizada en grupos pequeños y se trató luego de promover discusiones generales a partir de puntos problemáticos, seguida de eventuales exposiciones de los profesores tendientes a aclarar y profundizar los contenidos de la disciplina y a propiciar la reflexión metacognitiva.

A continuación se muestran ejemplos de las actividades que se desarrollaron durante el curso. En cada caso se explicitan los objetivos pretendidos y se analizan y evalúan los resultados obtenidos.

\section{Actividad 3 (Presencial y grupal)}

a) Con el objeto de identificar contenidos conceptuales, procedimentales y actitudinales, analizar el texto siguiente, preparado para ser usado en una primera clase sobre fenómenos térmicos:

«Las intensidades de las sensaciones táctiles que nos producen los cuerpos son susceptibles de gradación. Las palabras caliente, tibio, frío, helado expresan una serie de sensaciones correspondientes a diversos estados de un cuerpo. Sin embargo, estas sensaciones pueden, en ciertas circunstancias, inducirnos a error.

A1. Mencionar algunas experiencias para comprobar que el sentido del tacto puede llevarnos a falsas conclusiones.

A2. Proponer algún fenómeno físico que pueda ser utilizado para construir un instrumento de medición que permita caracterizar el estado térmico de los cuerpos.

Es sabido que los alumnos tienen dificultades para diferenciar los conceptos de calor y temperatura. A conti- 
Tabla III

Contenidos básicos comunues (fenómenos térmicos).

\begin{tabular}{|c|c|c|c|}
\hline & EGB & \multicolumn{2}{|c|}{ EDUCACIÓN POLIMODAL } \\
\hline & 3r. ciclo & Formación general de fundamento & $\begin{array}{l}\text { C. Básicas orient. } \\
\text { Modalidad ciencias naturales, } \\
\text { salud y ambiente }\end{array}$ \\
\hline 兽 & $\begin{array}{l}\text { - Equilibrio térmico } \\
\text { - Escalas de temperatura } \\
\text { - Equivalente entre calor y } \\
\text { trabajo }\end{array}$ & $\begin{array}{l}\text { - Fenómeno de transmisión de calor. Modelo } \\
\text { cinético de los gases. Principios de la termodiná- } \\
\text { mica. Nociones de irreversibilidad y entropía. } \\
\text { Aplicaciones al análisis de fenómenos naturales } \\
\text { y de procesos tecnológicos. }\end{array}$ & $\begin{array}{l}\text { - Equivalencia entre diversas formas de ener- } \\
\text { gía. Conversión de energía y disipación. As- } \\
\text { pectos termodinámicos: sistemas cerrados y } \\
\text { abiertos. Segundo principio de la termodiná- } \\
\text { mica. Entropía. }\end{array}$ \\
\hline 至 & $\begin{array}{l}\text { - Interpretación de escalas } \\
\text { de temperatura y traducción } \\
\text { de unas a otras } \\
\text { - Diseño y desarrollo de ex- } \\
\text { perimentos para medir la pér- } \\
\text { dida de calor por radiación }\end{array}$ & $\begin{array}{l}\text {-Diseño y realización de experimentos acerca de } \\
\text { procesos de conversión almacenamiento y flujo } \\
\text { de energía. } \\
\text {-Análisis de los fenómenos térmicos involucra- } \\
\text { dos en el funcionamiento de objetos tecnológi- } \\
\text { cos o sistemas naturales. } \\
\text {-Análisis de experimentos históricos en el cam- } \\
\text { po de la física relacionados con las temáticas } \\
\text { estudiadas. }\end{array}$ & $\begin{array}{l}\text { - Cálculo del balance energético de un siste- } \\
\text { ma empleando principios termodinámicos. }\end{array}$ \\
\hline
\end{tabular}

nuación, se proponen algunas actividades que pueden realizarse en el aula, con el objeto de discriminar estos conceptos.

A3. Idear alguna experiencia a través de la cual compruebe que la temperatura de un cuerpo (que puede medir con un termómetro) no es manifestación del calor que se le ha transferido al cuerpo.

A4. Formular hipótesis sobre los factores que determinan cuánto aumenta la temperatura de, por ejemplo, una determinada cantidad de agua, contenida en un recipiente en contacto con una llama o sobre un calentador. Hacer hipótesis sobre la relación que existe entre el calor transmitido al cuerpo (o por el cuerpo) y los cambios de temperatura que experimenta.

A5. Idear algún dispositivo experimental para contrastar sus hipótesis y proceder a su comprobación o falsación.

A6. Tratar de expresar matemáticamente los resultados cualitativos obtenidos en A5. Explicitar el significado físico de cada uno de los términos que intervienen en la expresión matemática propuesta e indicar, además, las unidades de medida de las cantidades físicas representadas por dichos términos.
Nota: Al realizar la pequeña investigación en el aula, se producen algunos errores que afectan los resultados numéricos. Sin embargo, la relación obtenida cualitativamente es valiosa, por cuanto brinda al alumno la posibilidad de discriminar los conceptos de calor y temperatura en forma empírica a través de una actividad motivadora. Es importante en este momento hacer notar el valor predictivo de la relación matemática encontrada en A6. Para ello, se recomienda realizar ejercicios y problemas de aplicación».

b) Las actividades $A l$ a $A 6$, ¿se adecúan, a su criterio, al modelo reticular de aprendizaje propuesto por Duschl?

c) Evaluar posibles aspectos positivos y negativos para la utilización del texto en el aula.

Actividad 5 (Presencial y grupal)

Leer críticamente el capítulo 19 del libro Fundamentos de la física moderna, de Holton y Roller.

Advertencia: Este texto corresponde a una edición de 1963. En los más de treinta años transcurridos, han continuado refinándose los conceptos y también la terminología. 
Guía de trabajo

a) Comprobar si la evolución histórica de los conceptos vinculados a los fenómenos térmicos puede ser explicada en términos de la red triádica que propone Laudan para el desarrollo del conocimiento científico.

b) Analizar la terminología utilizada por los científicos citados en el texto (Black, Rumford, etc.), para:

- establecer las diferencias con la aceptada hoy en día por la comunidad científica;

- identificar los términos que aún se utilizan en física (y en la vida diaria) y que favorecen la persistencia de concepciones y modelos ya superados en la ciencia.

c) Evaluar la conveniencia de incorporar (o no) información de tipo histórico en el desarrollo de estos temas en el aula. (Analizar para diferentes niveles educativos.)

d) Hacer un estudio comparativo de las etapas que fueron necesarias para lograr el cambio de paradigma (calórico $\rightarrow$ energía asociada al movimiento molecular) y las etapas necesarias para que los estudiantes logren el correspondiente cambio conceptual desde sus ideas intuitivas hacia el conocimiento científico.

e) Hacer una síntesis para discutir colectivamente.

\section{Actividad 8}

\section{Primera parte}

Esta actividad propone la construcción de un colector solar rudimentario utilizando tres chapas metálicas (dos negras y una blanca), con áreas aproximadamente iguales, una placa de vidrio de área mayor que las de las chapas, un trozo de material aislante (telgopor, o algodón o lana) y un soporte de madera.

La estructura de los materiales apropiados para la construcción se determina al investigarse la absorción de la luz por superficies de colores diferentes y procesos como la conducción, convección y radiación térmicas.

Procedimiento y preguntas:

1) Coloque dos chapas metálicas (una negra y una blanca) expuestas al sol, fijando previamente un termómetro en cada una de ellas. Lea y anote la variación de la temperatura con el tiempo, en cada chapa. En intervalos de tiempo iguales, ¿cuál de ellas alcanza mayor temperatura?

2) Coloque agua en dos tubos de aproximadamente $2 \mathrm{~cm}$ de diámetro y $20 \mathrm{~cm}$ de altura cada uno, siendo uno de ellos metálico y el otro de plástico o PVC, del mismo color.

Expóngalos al sol durante el mismo intervalo de tiempo anotando la temperatura del agua antes y después de la exposición. Retire los tubos del sol y verifique en cuál de ellos la temperatura del agua es mayor. ¿Cómo explica este hecho?
3) Exponga al sol dos chapas metálicas negras, cada una con un termómetro, de la misma forma que en el ítem 1. Encima de una de ellas, aproximadamente a $2 \mathrm{~cm}$, coloque una placa de vidrio. Ahora anote el tiempo que permanecen expuestas al sol y las indicaciones de los termómetros en ambas placas. ¿En cuál de ellas la temperatura es mayor? ¿Qué papel atribuye al vidrio?

4) Debajo del montaje de la chapa y el vidrio coloque una placa de material aislante. Exponga el conjunto nuevamente al sol durante el mismo intervalo de tiempo de la exposición anterior. Anote la temperatura alcanzada en esta situación y compárela con la que registró en el ítem 3. ¿Cómo explica la diferencia?

5) Considerando los resultados obtenidos en esta actividad, ¿cómo montaría un colector solar? Explique la función de cada uno de los componentes que utilizaría en el colector.

6) ¿Cómo puede mejorar la eficiencia del colector?

7) En la instalación de un colector solar, ¿dónde debe estar colocada la entrada de agua fría y la salida de agua caliente?

\section{Segunda parte}

a) Analice e identifique objetivos, métodos y teorías puestos en juego en la tareas propuestas.

b) Analice si las observaciones realizadas se ajustan al modelo de observación tripartito de Shapere. Explicite las teorías acerca de la fuente, la transmisión y el receptor en las observaciones realizadas.

c) Elabore en grupo una breve síntesis de las respuestas y las conclusiones logradas respecto a toda la actividad.

Algunos comentarios sobre las actividades:

Cada uno de los ejemplos mostrados tuvo un objetivo distinto. La actividad 3 fue diseñada especialmente para desarrollar contenidos procedimentales. Como es sabido, esto sólo puede lograrse a través del empleo de contenidos conceptuales (Coll et al., 1994), por lo que se debió profundizar previamente los conceptos físicos involucrados.

Los ítems $b$ y $c$ de la actividad 3 requirieron una reflexión sobre la pertinencia de la propuesta de Duschl y de su adecuación a las necesidades y características de alumnos de los diferentes niveles educativos.

Uno de los principales obstáculos encontrados durante la discusión grupal fue la ambigüedad de los términos científicos usados en la termodinámica, tal como lo señalan Vicentini y Mayer (1996).

La actividad 5 tiene múltiples objetivos: el análisis de experimentos históricos, la revisión de la evolución de las teorías, destacar la importancia de los métodos y nuevos instrumentos en el desarrollo y cambio de teo- 
rías, para así destacar cómo la interconexión entre la ciencia, la tecnología y los requerimentos de la sociedad conduce al progreso de la propia ciencia.

En particular, se pretendía reafirmar el paralelismo entre el cambio de teorías en la ciencia (progreso) y el cambio conceptual que se pretende en los alumnos (aprendizaje). En síntesis, los objetivos fueron de carácter epistemológico, científico, metodológico, psicológico y metacognitivos.

La actividad 8, de carácter experimental, fue pensada como una actividad de síntesis, pues en ella, además de los objetivos perseguidos en las actividades antes descritas, se buscaba realizar una contrastación de la pertinencia del empleo del proceso tripartito de observación de Shapere como referente teórico para la selección y planificación de tareas para el aula.

\section{EVALUACIÓN DE LA EXPERIENCIA}

Los participantes eran todos docentes. El 30\% eran alumnos del magister, y los restantes, profesores de física en ejercicio para quienes las exigencias para la aprobación del curso fueron menores. Los participantes constituyeron un grupo que, en su práctica docente, cubrían desde el ciclo EGB 3 hasta el nivel universitario básico.

Para evaluar la adecuación de los referentes teóricos a los objetivos del curso, como así también el desarrollo del mismo y la participación y aceptación de las propuestas por parte de los participantes, durante su realización, hemos alternado nuestros roles de coordinador y de observador a fin de realizar una investigación exploratoria en la que se utilizaron, para la recolección de datos, técnicas etnográficas como la observación participante, complementada con cuestionarios escritos semiestructurados y entrevistas abiertas.

Como recurso metodológico para asegurar la validez de los datos, no sólo alternamos los roles, sino que tomamos registros simultáneos, lo que permitió la comparación y contrastación de interpretaciones con el fin de minimizar subjetividades.

Realizamos estudios por observación, pues permiten incorporar datos provenientes de comportamientos no verbales, que generalmente no están ni sesgados ni polarizados.

En general, los alumnos consideraron importantes y valiosos los aportes epistemológicos que fundamentan el diseño didáctico, salvo un docente que parecía sólo interesado en los aspectos experimentales de las actividades, ya que sostenía que este tipo de tareas es el único que motiva a los estudiantes adolescentes. Esta actitud, que mantuvo durante todo el curso, generó rechazo en el resto de los participantes.

Los grupos de trabajo se constituyeron espontáneamente y conforme al nivel en el cual los participantes desarro- llaban su práctica docente. Esto resultó una ventaja, puesto que cada grupo evaluó las potencialidades de cada actividad, y de la propuesta global y su marco teórico para su posterior transferencia al aula. Asimismo se enriqueció la discusión en las sesiones plenarias, ya que hubo intercambio de experiencias, diferentes niveles de análisis y explicitación de problemáticas características de cada ciclo del sistema educativo.

A partir de los registros de las observaciones y de la información obtenida mediante las entrevistas informales realizadas, categorizamos comportamientos y respuestas, lo que nos permitió efectuar una valoración favorable del diseño instruccional para la enseñanza de los fenómenos térmicos, por los aspectos que pasamos a enumerar:

1) La red triádica resultó ser una herramienta útil para seleccionar y secuenciar los contenidos y las actividades.

2) A través de la red, los participantes integraron los distintos tipos de contenidos, según el «principio de nivelación» mencionado por Laudan. Les permitió valorar la importancia de tener en cuenta, en la etapa de planificación de actividades, la explicitación de teorías, métodos y objetivos.

3) El proceso tripartito de observación de Shapere también resultó fructífero a la hora de analizar los fenómenos, como, por ejemplo, los de transferencia de energía entre sistemas.

4) El análisis histórico en el que se puso en evidencia el carácter dinámico de la ciencia permitió que muchos de los participantes tomaran conciencia de la magnitud de las dificultades que representa para los alumnos el abandono de sus «confortables» concepciones previas.

5) También el análisis del desarrollo de los conceptos científicos (en particular, calor) puso de manifiesto que el lenguaje científico es en algunos aspectos ambiguo. Igualmente se destacó la importancia que tiene el uso, por parte de los docentes, de un lenguaje adecuado y cuidadoso, durante el desarrollo de las clases para evitar reforzar las ideas previas de los alumnos.

Se realizó también una evaluación final del curso mediante una encuesta escrita semiestructurada en la que la totalidad de los asistentes expresó su convicción de que la propuesta es aplicable en sus respectivos ámbitos de trabajo, con la condición previa de profundizar y completar el estudio de los fundamentos epistemológicos, los cuales eran poco conocidos por la mayoría de los participantes.

\section{CONCLUSIONES}

Consideramos que el marco teórico adoptado a partir de la propuesta de Duschl resulta muy adecuado para res- 
ponder a los requerimientos, no sólo de la reforma educativa de la enseñanza media, sino que también es válido y recomendable para la docencia en el nivel universitario.

La jerarquía que el modelo permite brindar a los contenidos procedimentales y a los objetivos y valores nos parece uno de sus aspectos más relevantes, por cuanto consideramos que el vertiginoso avance y desarrollo del conocimiento científico hace necesario que los estudiantes adquieran competencias sobre todo de carácter procedimental. El desarrollo y afianzamiento de las destrezas cognitivas serán los que posibiliten una rápida adaptación a los cambios que se produzcan en el dominio conceptual.

Queda pendiente analizar si el marco teórico adoptado en este curso -exitosamente según la valoración hecha

\section{REFERENCIAS BIBLIOGRÁFICAS}

BANDIERA, M., DUPRÉ, F., IANNIELLO, M.G. y VICENTINI, M. (1995). Una investigación sobre las habilidades para el aprendizaje científico. Enseñanza de las Ciencias, 13(1), pp. 46-54.

COLL, C. et al. (1994). Los contenidos en la reforma. Buenos Aires: Santillana.

DUSCHL, R. (1997). Renovar la enseñanza de las ciencias. Madrid: Narcea, SA.

DUSCHL, R. (1998). El valor de las argumentaciones y las explicaciones: promover estrategias de retroalimentación. Enseñanza de las Ciencias, 16(1), pp. 3-20.

GIL PÉREZ, D. (1986). La metodología científica y la enseñanza de las ciencias. Unas relaciones controvertidas. Enseñanza de las Ciencias, 4(2), pp. 111-121. por los participantes-es igualmente útil para la toma de decisiones en la implementación de cursos de física en otros niveles educativos y en otros campos de la física.

Esperamos que a partir de su participación como alumnos del curso, y en función de los logros alcanzados en este rol, los participantes puedan transferir a su práctica docente el marco teórico y la metodología usados en el transcurso de esta experiencia.

\section{NOTA}

Una versión preliminar de este trabajo fue presentada como ponencia en el IV Simposio de Investigadores en Educación en Física. Setiembre de 1998. La Plata. Argentina.
LAUDAN, L. (1977). Progress and its problems. Berkeley, CA: University of California Press.

LAUDAN, L. (1984). Science and Values. Berkeley, CA: University of California Press.

POSNER et al. (1982). Accomodation of a scientific conception: Toward a theory of conceptual change. Science Education, 66, pp. 211-227

SHAPERE, D. (1982). The concept of observation in science and philosophy. Philosophy of Science, 59, pp. 485-525. Citado en Duschl, R. (1997).

VICENTINI, M. y MAYER, M. (1996). Didattica della Fisica. Florencia: La Nuova Italia Ed.

VILLANI, A. (1992). Conceptual change in Science and Science Education. Science Education, 76(2), pp. 223-237.

[Artículo recibido en diciembre de 1998 y aceptado en mayo de 1999.] 